\title{
Erratum to: Association between osteocalcin and glucose metabolism: a meta-analysis
}

\author{
D.-M. Liu ${ }^{1,2}$ - X.-Z. Guo ${ }^{1,2}$ - H.-J. Tong ${ }^{3}$ - B. Tao ${ }^{1,2} \cdot$ L.-H. Sun ${ }^{1,2} \cdot$ H.-Y. Zhao ${ }^{1,2}$ • \\ G. Ning ${ }^{1,2} \cdot$ J.-M. Liu ${ }^{1,2}$
}

Published online: 14 September 2015

(C) International Osteoporosis Foundation and National Osteoporosis Foundation 2015

\section{Erratum to: Osteoporos Int}

\section{DOI 10.1007/s00198-015-3197-8}

Panel $\mathbf{a}$ of Fig. 3 and the accompanying legend were incorrect. The authors and the publisher apologize for this error and regret any inconvenience caused. The amended figure is given here.

\section{G. Ning}

guangning@medmail.com.cn

$\triangle$ J.-M. Liu

ljmhh@sh163.net

1 Department of Endocrine and Metabolic Diseases, Rui-jin Hospital, Shanghai Jiao-Tong University School of Medicine, Shanghai, China

2 Shanghai Institute of Endocrine and Metabolic Diseases, Shanghai, China

3 Institut de Recherches Cliniques de Montréal (IRCM) Department of Microbiology and Immunology, University of Montreal and Division of Experimental Medicine, McGill University, Montreal, QC H2W 1R7, Canada 
Fig. 3 a, b Correlation $(95 \% \mathrm{CI})$ between tOcn (a) or unOcn (b) and $\mathrm{HbAl} \mathrm{c}$ from a fixed-effects model and a random-effects model, respectively a

$\mathrm{ES}(95 \% \mathrm{CI})$

$\%$

Im JA,2008

Zhou M,2009

Zhang Y,2010

Bae SJ,2011(Men)

Bae SJ,2011(Postmenopausal)

Bao YQ,2011a

Bao YQ,2011b

Kanazawa I,2011(Men)

Kanazawa I,2011(Postmenopausal)

Iki M,2012

Alfadda AA,2013

Bao YQ,2013

Buday B,2013(Men)

Buday B,2013(Women)

Dou J,2013

Ogawa-Furuya N,2013(Men)

Ogawa-Furuya N,2013(Postmenopausal)

Schwetz V,2013

Sheng L,2013

Wang QQ,2013

Weiler HA,2013

Cui R,2014

Maddaloni E,2014

Rui XF,2014

Overall $(\mathrm{I}-\mathrm{squared}=43.1 \%, \mathrm{p}=0.014)$
$-0.22(-0.33,-0.12)$

$-0.09) \quad 4.83$

$-0.09(-0.19,-0.00) \quad 4.45$

$-0.28(-0.42,-0.14) \quad 1.89$

$-0.24(-0.35,-0.14) \quad 3.56$

$-0.22(-0.37,-0.08) \quad 1.73$

$-0.33(-0.59,-0.07) \quad 0.54$

$-0.21(-0.36,-0.07) \quad 1.72$

$-0.20(-0.39,-0.01) \quad 1.03$

$-0.14(-0.19,-0.09) \quad 15.49$

$-0.20(-0.34,-0.06) \quad 1.94$

$-0.17(-0.22,-0.12) \quad 17.15$

$-0.09(-0.25,0.07) \quad 1.48$

$-0.06(-0.23,0.11) \quad 1.28$

$-0.12(-0.17,-0.07) \quad 15.11$

$-0.17(-0.36,0.01) \quad 1.12$

$-0.15(-0.35,0.05) \quad 0.94$

$-0.14(-0.30,0.02) \quad 1.45$

$-0.02(-0.09,0.04) \quad 7.91$

$-0.28(-0.53,-0.04) \quad 0.61$

$-0.23(-0.34,-0.13) \quad 3.55$

$-0.21(-0.41,-0.01) \quad 0.92$

$-0.09(-0.30,0.11) \quad 0.87$

$-0.24(-0.31,-0.16) \quad 7.15$

$-0.16(-0.18,-0.14) \quad 100.00$ ight

4.83
4.5
.89
3.56
.73
0.54
.72
1.3
.49
1.94
1.48
.28
11
.12
0.94
7.91
0.61
3.55
0.92
87
7.15
100.00

$-.59$

1
.59 